\title{
A GRADIENT BOUND FOR THE GRAD-KRUSKAL-KULSRUD FUNCTIONAL
}

\author{
Peter Laurence And Edward Stredulinsky
}

\begin{abstract}
A вsтRACт. We announce a gradient bound for minimizers of a variational problem arising in the Grad-Kruskal-Kulsrud model for the equilibrium of a confined plasma. The variational problem involves derivatives of the nondecreasing rearrangement of minimizers. In the case of a convex domain our results answer in the negative a question raised by Grad concerning the possibly singular behavior of the magnetic field at the point of maximum flux. It completes a research program begun in 1983 in which an approach using free boundaries was initiated to handle the nonlinear nonlocal nature of the problem. Limiting minimizers of the variational problem are shown to have bounded gradient and satisfy a weak equation which for one model problem takes the form
\end{abstract}

$$
\triangle \psi=-\psi^{* \prime \prime}\left(\mu_{\psi}(\psi)\right)
$$

where $\psi^{*}, \mu_{\psi}$ are respectively the nondecreasing rearrangement, and distribution function of $\psi$.

\section{Plasmas and variational problems with global constraints}

The Grad-Kruskal-Kulsrud model (hereafter GKK) [GHS], [KK] for the confinement of a thermonuclear plasma is a realistic one in which the nonlinearities are nonlocal nonlinear functionals of the solution. Here we apply the variational problem to axisymmetric magnetic bottles, known as tokamaks $[\mathrm{CH}]$. These devices are at the forefront of experimental research today. [TFTR at Princeton, Jet at Culham, ...]. Associated with the plasma in equilibrium is an energy, expressed in terms of the magnetic field $B$

1991 Mathematics Subject Classification. Primary 35r35, 35d10, 34jxx; Secondary $35 \mathrm{q} 35$.

Key words and phrases. Grad functional, free boundary problem, gradient bound. Received December 14, 1993.

E. Stredulinsky partially supported by NSF Grant DMS-9102886. 
and the scalar pressure $p$, which must be minimized over an appropriate function space:

$$
E=\int_{T} \frac{|B|^{2}}{2}+p
$$

In order to deal with the nonlocal and nonlinear structure of the variational problem a method of approximation via a sequence of free boundary problems was introduced in [LS1]. Although the method soon proved powerful enough $[\mathrm{LS} 4,5]$ to yield a gradient bound for certain semilinear elliptic problems with nonmonotone, $L^{1}$ nonlinearities, its application to the Grad variational problem proved substantially more challenging and for a long period produced only partial results. Over the last year the method was further refined so that it could be applied successfully to the Grad functional. The purpose of the present announcement is to advertise the coming of age of this method along with its successful application to a problem that has resisted other approaches.

In this announcement for simplicity we will, following Grad, consider a simplified model where curvature effects are ignored and the axisymmetric torus is replaced by a periodic cylinder. As in the axisymmetric case the variational problem then reduces to one in the cross-section of the cylinder. The magnetic field may then be represented as the orthogonal gradient of a stream function $\psi$ and $p$ may be eliminated in favor of the density $\rho$ assuming an adiabatic equation of state, i.e.

$$
B=\nabla^{\perp} \psi(x)+B^{z}(x) \nabla z, \quad p=S \rho^{2}
$$

where the $z$ axis is orthogonal to the cylindrical cross-section $\Omega, B^{z}$ is the $z$ component of the magnetic field, $\nabla z$ is a unit vector in the $z$ direction, and the constant $\mathrm{S}$ is the entropy. The energy (1) can then be written

$$
J(\psi)=\int_{\Omega}\left\{|\nabla \psi|^{2}+\left|B^{z}\right|^{2}+S \rho^{2}\right\} d A
$$

where $d A$ denotes integration with respect to Lebesgue measure in the cross-section $\Omega$. The energy (2) is to be minimized subject to constraints of prescribed total mass $M(\psi)$ within the flux surfaces $\{\psi=c\}$ and prescribed total toroidal flux $\mu(\psi)$ defined by

$$
M(c)=\int_{\psi>c} \rho d A, \quad \mu(c)=\int_{\psi>c} B \cdot \nabla z d A .
$$


The constraints are

$$
M(\psi)=f_{1}(\psi), \quad \mu(\psi)=f_{2}(\psi),
$$

where $f_{1}$ and $f_{2}$ are given continuous functions and $f_{1}$ is positive.

Solutions are normalized by setting $\psi=0$ at $\partial \Omega$, and the total flux $\max _{\Omega} \psi-\min _{\Omega} \psi$ is normalized to one.

It was shown heuristically in [KK] and rigorously in [LS6] that the functional $J(\psi)$ achieves its minimum, subject to the constraints and $\left.\psi\right|_{\partial \Omega}=0$, at a function $\psi$ which minimizes the Grad functional

$$
\left.J(\psi)=\int_{\Omega}|\nabla \psi|^{2} d A+\int_{0}^{|\Omega|}\left[S{f_{1}^{\prime 2}}^{\prime 2} \psi\right)+{f_{2}^{\prime 2}}^{2}(\psi)\right] \psi^{*^{2}}(\mu) d \mu
$$

where $\psi^{*}$ is the nondecreasing rearrangement of $\psi$ defined by

$$
\psi^{*}(\mu)=\sup \{t:|\{x: \psi(x)<t\}|<\mu\} .
$$

Formula (5) is the functional studied in [GHS]. The variational problem was studied by Laurence and Stredulinsky [LS1-3] after Mossino and Temam had introduced a slightly different version of the problem to the mathematical community in [MT]. For simplicity, take $S=1, f_{1}(t)=$ $f_{2}(t)=t / 2$ to obtain the model problem

$$
\widetilde{J}(\psi)=\int_{\Omega}|\nabla \psi|^{2} d A+\int_{0}^{|\Omega|} \psi^{\prime^{2}}(\mu) d \mu
$$

with the Euler equation:

$$
\Delta \psi=-\psi^{* \prime \prime}\left(\mu_{\psi}(\psi)\right),
$$

where $\mu_{\psi}(t)=|\{\psi>t\}|$ is the distribution function of $\psi$. In general there is no simple expression for $\psi^{* \prime \prime}$. If $|\nabla \psi|$ is bounded from zero and $\psi$ is smooth one has

$$
\psi^{* \prime \prime}\left(\mu_{\psi}(t)\right)=\left\{\int_{\psi=t} \nabla \cdot\left(\frac{\nabla \psi}{|\nabla \psi|^{2}}\right) \frac{d \ell}{|\nabla \psi|}\right\} \psi^{* \prime^{3}} .
$$

The functional $\widetilde{J}$ is to be minimized on the space

$$
W=\left\{\psi \in H_{0}^{1}(\Omega): \psi^{*} \in H^{1}(0,|\Omega|), \psi^{*}(0)=0, \psi^{*}(|\Omega|)=1\right\}
$$




\section{Results}

We have obtained a gradient bound for certain minimizers of $\tilde{J}$ on $W$ when $\Omega$ is a convex subset of $\Re^{2}$. Uniqueness is not known so we cannot claim a gradient bound for all minimizers. Our method, which is constructive, approximates the variational problem by a sequence of free boundary problems. The $n^{\text {th }}$ approximating free boundary problem is associated with $n$-layers $A_{i}^{(n)}=\left\{x: t_{i-1}^{(n)} \leq \psi_{n}<t_{i}^{(n)}\right\}, i=1, \ldots, n-1$, $A_{n}^{(n)}=\left\{\psi_{n}=t_{n-1}^{(n)}\right\}$ (minimizers satisfy $\inf \psi_{n}=0, \sup \psi_{n}=t_{n-1}^{(n)}$ ), with its minimizers $\psi_{n}$ being harmonic within each layer and satisfying a jump condition

$$
\left|\nabla \psi_{n}^{\text {ext }}\right|^{2}-\left|\nabla \psi_{n}^{\text {int }}\right|^{2}=\left(\frac{\Delta t_{i+1}^{(n)}}{\left|A_{i+1}^{(n)}\right|}\right)^{2}-\left(\frac{\Delta t_{i}^{(n)}}{\left|A_{i}^{(n)}\right|}\right)^{2}
$$

across the free boundaries $\Gamma_{i}^{(n)}=\partial\left\{\psi_{n}=t_{i}^{(n)}\right\}, i=1, \ldots, n-2$. Here $\left|A_{i}^{(n)}\right|$ denotes the Lebesgue measure of $A_{i}^{(n)}$, and $0=t_{0}^{(n)}<t_{1}^{(n)}<\ldots<t_{n}^{(n)}=1$, $\triangle t_{i}^{(n)}=t_{i}^{(n)}-t_{i-1}^{(n)}$. Due to an area constraint on $A_{n}^{(n)}$ the gradient jump across $\Gamma_{n-1}^{(n)}=\partial\left\{\psi_{n}=t_{n-1}^{(n)}\right\}$ is an unknown constant which can only be bounded using an inequality analogous to (8).

The key to our approach is to obtain a gradient bound, uniform in $n$, on minimizers of the approximating problems. This is combined with a proof that the minimizers of the approximating problems converge to a minimizer of $(7)$.

Our efforts to obtain a gradient bound for minimizers of (7) were initiated in [LS1]. From the outset we focused, as we also do here, on the case where $\Omega$ is convex. However a fundamental difference is that in earlier work [LS1,3] an extraneous assumption was made that level sets of minimizers were to be convex. The gradient bound we present here is independent of this or any other extraneous assumption.

An indication as to why one may expect a gradient bound for minimizers of $\widetilde{J}$ comes from the following a priori estimate derived in [LS1]. By making a range variation in $\widetilde{J}$ one obtains

$$
\psi^{* \prime}+\int_{\{\psi=t\}}|\nabla \psi|=\inf \widetilde{J}
$$

thus $\psi^{* \prime}$ is bounded by the minimum energy. Also as follows by a $p$ function 
argument of Mossino [M], and Payne and Stakgold [PS]

$$
P(\psi)=|\nabla \psi|^{2}(x)+\int_{0}^{\psi(x)} \psi^{* \prime \prime}\left(\mu_{\psi}(t)\right) d t
$$

attains its maximum where $\nabla \psi=0$. Thus introducing a change of variables $t=\psi^{*}(\mu)$ in (10) and integrating one gets in conjunction with (9) the inequality

$$
|\nabla \psi| \leq \psi^{* \prime}\left(\mu_{\psi}(\psi(\bar{x}))\right) \leq \inf \widetilde{J}
$$

The method of $[\mathrm{M}],[\mathrm{PS}]$ assumes $\psi \in C^{2}$ and $\psi$ is superharmonic. As minimizers are only in $H_{0}^{1}$ the point of using the approximating variational problems is to find a version of (11) (and (9)) that can be established in the approximating setting where minimizers are Lipschitz continuous. Also our proof does not rely on superharmonicity (which is not known at this point). One then passes to the limit to obtain a gradient bound for a minimizer of (7). Our main result is

Theorem 1. If $\Omega$ is convex then there exists a Lipschitz continuous minimizer $\psi$ of $\widetilde{J}$ on $W$ satisfying

$$
|\nabla \psi| \leq \inf \widetilde{J} \text { a.e. }
$$

This minimizer satisfies the following weak form of the Euler equation

$$
\begin{array}{r}
\int_{\Omega}\left\{(\nabla \psi)^{\perp}\left[(\nabla \psi)^{\perp} \cdot \nabla \eta\right]+\left[\frac{1}{2}|\nabla \psi|^{2}+{\psi^{* \prime^{2}}}\left(\mu_{\psi}(\psi)\right)\right] \nabla \eta\right\} d A=0 \\
\forall \eta \in C_{0}^{\infty}(\Omega) \times C_{0}^{\infty}(\Omega) .
\end{array}
$$

The weak equation (13) is derived in [LS6] directly by making appropriate variations without the aid of the approximating problems. On the other hand equation (12) will require the full strength of the approximating free boundary problems which we now introduce.

\section{Approximating free boundary problems}

Let

$$
J_{T}(\psi)=\int_{\Omega}|\nabla \psi|^{2} d A+\sum_{i=1}^{n} \frac{\Delta t_{i}^{2}}{\left|A_{i}\right|}
$$


where $T=\left\{t_{0}=0<t_{1}<\cdots<t_{n}=1\right\}$ is a partition of the interval $[0,1], A_{i}=\left\{t_{i-1} \leq \psi<t_{i}\right\}$, and where $\Gamma_{i}=\partial\left\{\psi=t_{i}\right\}, i=1, \ldots, n-2$, $\Gamma_{n-1}=\partial\left\{t_{n-1} \leq \psi\right\}$ are the $n-1$ free boundaries corresponding to the partition $T$. Consider the approximating variational problem of finding $a=a_{n} \in(0,|\Omega|)$ at which the following supremum is attained

$$
S_{n}=\sup _{0<a<|\Omega|} \inf _{(T, \psi) \in W_{a, n}} J_{T}(\psi)
$$

where

$$
\begin{aligned}
& W_{a, n}=\left\{(T, \psi): T=\left\{t_{0}=0<t_{1}<\cdots<t_{n}=1\right\}, \triangle t_{n} \in(0,1),\right. \\
& \left.\triangle t_{1}=\cdots=\triangle t_{n-1}, \psi \in H_{0}^{1}(\Omega),\left|A_{n}\right|=\left|\left\{t_{n-1} \leq \psi<t_{n}\right\}\right|=a\right\},
\end{aligned}
$$

and a pair $\left(T^{(n)}, \psi_{n}\right)$ at which the inf in (15) is attained for $a=a_{n}$ i.e. $S_{n}=J_{T^{(n)}}\left(\psi_{n}\right)$. Formally the functions $\psi_{n}$ satisfy condition (8) at the free boundaries $\Gamma_{i}^{(n)}$. We refer to this problem as the approximating GradKruskal-Kulsrud problem (AGKK). A natural question is to ask why we need the area constraint in $W_{a, n}$ and the supremum over $a$ in (15). The answer is that to establish a uniform gradient bound one needs an analogue of (9) in the free boundary setting. But (9) was established using a range variation. It turns out that the only straightforward way to do a range variation in the free boundary setting is to allow variation in the levels $t_{i}, i=1, \ldots, n-1$, while fixing $a_{n}=\left|A_{n}^{(n)}\right|$ to prevent the minimum of the functional from degenerating to zero. Unfortunately one then loses knowledge of the magnitude of the jump in the gradient at the innermost free boundary (an area constraint implies only that the jump is constant) which prevents a complete gradient bound from being established. However an estimate of the gradient jump can be recovered for the special value of $a$ for which the supremum in (15) is obtained.

Theorem 2. Let $\Omega$ be convex. Then there exists a Lipschitz continuous function $\psi_{n}$ which solves $A G K K$ and satisfies

$$
\left|\nabla \psi_{n}\right| \leq \Delta t_{n}^{(n)} /\left|A_{n}^{(n)}\right| \quad \text { a.e. }
$$

and

$$
\Delta t_{n}^{(n)} /\left|A_{n}^{(n)}\right| \leq J_{T}\left(\psi_{n}\right) \leq \inf _{W} \widetilde{J}
$$


The proof of Theorem 2 relies on a maximum principle for $|\nabla \psi|$ which circumvents the difficulty that $\nabla \psi$ may not be defined in a classical sense at points on the free boundaries $\Gamma_{i}$ which are elements of an exceptional set $\mathcal{C}$ of zero one dimensional Hausdorff measure. The free boundary condition (8) is satisfied classically off of $\mathcal{C}$ and in addition for $x \in \mathcal{C}$ in the following weak sense:

$$
\limsup _{\substack{y \rightarrow x \\ y \in A_{i}}}\left|\nabla \psi_{n}(y)\right|^{2}+\left(\frac{\Delta t_{i}}{\left|A_{i}\right|}\right)^{2}=\limsup _{\substack{y \rightarrow x \\ y \in A_{i+1}}}\left|\nabla \psi_{n}(y)\right|^{2}+\left(\frac{\Delta t_{i+1}}{\left|A_{i+1}\right|}\right)^{2}
$$

Theorem 3. (Limiting Behavior). The solutions $\psi_{n}$ of AGKK satisfy (i) $\left|A_{n}^{(n)}\right| \rightarrow 0,($ ii $) \psi_{n_{k}} \longrightarrow \psi$ in $H_{0}^{1}(\Omega)$ (for some subsequence $n_{k}$ ), where $\psi$ minimizes (7) over $W$.

That the limiting function $\psi$ in Theorem 3 is an element of $W$ is due to the fact that the summation term in (14) is the square of the $L^{2}$ norm of the derivative of the linearization of $\psi_{n}^{*}$ at levels $t_{i}^{(n)}$. This fact allows one to take advantage of the lower semicontinuity properties of the individual terms in the functional (7). Since the distribution functions of $\psi_{n}^{*}$ and its linearization agree at levels $t_{i}^{(n)}$, which are becoming dense in the limit, it is possible to establish that the linearizations of $\psi_{n}^{*}$ tend to $\psi^{*}$ in the limit and that the limiting function $\psi$ is a minimizer of (7) over $W$.

\section{Monotonicity formula mimics curvature}

If the free boundaries $\Gamma_{i}$ were known to be $C^{2}$, an argument involving their curvature, similar to that in [LS4], could be used to establish part i) of Theorem 2. In the presence of an exceptional set where the free boundaries are not smooth we can use the striking monotonicity formula in Alt, Caffarelli, Friedman $[\mathrm{ACF}]$ to mimic the effects of curvature at the exceptional points (see details in next section). The functional (14) is a nonlinear $n$ layer version of that in $[\mathrm{ACF}]$ (note $[\mathrm{BKP}]$ for a result on spherical caps which is needed in $[\mathrm{ACF}]$ ). Blow up arguments can be used to linearize our functional and recover crucial nondegeneracy estimates in $[\mathrm{ACF}]$.

\section{Outline of proofs}

In this section we explain the proof of Theorem 2, part $i$ ), which contains the application of the monotonicity formula. This part of the Theorem 
deals with regularity and a maximum principle. A first and fundamental step in our analysis consists in proving that the minimizers are Holder continuous. This first step uses Morrey's dyadic argument and is inspired by [ACF,Theorem 2.1 ]. It is more delicate however because of the presence in our case of the constraint $\left|\left\{\psi_{n} \geq t_{n}\right\}\right|=a$ which limits the variations that can be made. An immediate consequence of the continuity of our minimizers is that the free boundaries $\partial\left\{\psi_{n}=t_{i}\right\}, i=1, \ldots, n-1$ are separated (i.e. closed and nonintersecting) and so in our variational analysis we may vary one free boundary at a time. Thus for the most part we deal with a 2-phase free boundary problem. This two phase free boundary problem is a nonlinear version of that in $[\mathrm{ACF}]$ since, as noted before, the terms $\left|\left\{t_{i} \leq \psi_{n}<t_{i+1}\right\}\right|$ appear in the denominator rather than in the numerator.

We establish a maximum principle which shows that the sup of the modulus of the gradient of a solution of our n-layer free boundary problem (15) is bounded a.e. by the value of a quantity $\Lambda_{n}$ associated with $A_{n}=$ $\left|\left\{t_{n-1} \leq \psi_{n}<t_{n}\right\}\right|$.

We will refer to layer $A_{n}$ and the corresponding free boundary as 'innermost'. This terminology is introduced in order to make the ensuing discussion more visual. It has limited validity however since it is accurate only if the free boundaries $\partial\left\{\psi_{n}=t_{i}\right\}, i=1, \ldots, n$ are nested. At present we are unable to rule out the possibility that the free boundaries may have many components and that the level sets $\left\{\psi_{n}=t_{i}\right\}, i=1, \ldots, n-1$ may have positive measure (recall that the set $\left\{\psi_{n}=t_{n}\right\}$ has measure $a$ by construction). It turns out that the possible multiplicity of the components does not cause additional difficulties in our analysis except when dealing with the Haussdorff convergence of the innermost free boundaries associated with minimizing sequences.

The essence of our maximum principle is an argument based on the weak free boundary condition (16). Note that (16) is essentially equivalent to the statement that the definition of the function

$$
\Lambda(x)=|\nabla \psi(x)|^{2}+\frac{\triangle t_{i}^{2}}{\left|A_{i}\right|^{2}}, \quad \text { for } x \in A_{i}=\left\{t_{i-1} \leq \psi<t_{i}\right\}
$$

$i=1, \ldots, n$, can be extended to the closure of $\Omega \backslash A_{n}$ as an upper semicontinuous function. We will show that $\Lambda(x)$ attains its maximum on $\partial A_{n}$, this maximum being bounded by the value of $\Lambda$ on $A_{n}$. This leads to the 
inequality

$$
\sup _{\Omega} \Lambda=\sup _{A_{n}} \Lambda \geq \limsup _{\substack{x \rightarrow \partial\left\{\psi<t_{n-1}\right\} \\ \psi(x)<t_{n-1}}} \Lambda(x)
$$

from which our gradient bound is directly derived.

The derivation of the weak form of the free boundary condition requires the study of blow up limits of a minimizer at points on its free boundaries. These blow up limits are shown to be global minimizers of a linearized version of the functional (14). The linearization of (14) is essentially the functional in $[\mathrm{ACF}]$. In $[\mathrm{ACF}]$ blow up limits of the linearized functional have been shown to be either one or two plane solutions. This strong information when coupled with the monotonicity formula yields the weak free boundary condition (even in the linear case this seems to be new in the sense that it yields a useful free boundary condition at an earlier stage in the analysis). The monotonicity formula, in the two dimensional case, says that if $x_{0} \in\{u=0\}$ where $u$ is a function harmonic in $B_{R} \backslash\{u=0\}$ then the quantity

$$
M(u, r)=\frac{1}{\left|B_{r}\right|^{2}} \int_{B_{r}}\left|\nabla u^{+}\right|^{2} \cdot \int_{B_{r}}\left|\nabla u^{-}\right|^{2}
$$

is increasing in $r$ for small $r$.

To begin the proof of our gradient bound notice that $\Lambda(x)$ cannot reach its maximum on $\partial \Omega$. Indeed $\partial \Omega$ is convex and so by a well known identity [LS1, p. 353]

$$
\frac{\nabla \psi}{|\nabla \psi|} \cdot \nabla(|\nabla \psi|)=\kappa|\nabla \psi|
$$

( $\kappa$ is curvature of level curves of $\psi$ ) the gradient increases initially in the interior normal direction. In addition, since $g=|\nabla \psi|^{2}$ is subharmonic where $\psi$ is harmonic, $g$ can achieve an interior maximum in some $A_{i}$ only when constant. But from (17) this would imply that $g \equiv 0$ (which only happens in $A_{n}$ and so is not relevant in $\Omega \backslash A_{n}$ ), or $\kappa \equiv 0$, which only happens when level curves of $\psi$ are lines, i.e. $\psi$ is linear (due to constant gradient), a geometrical impossibility since $\Omega$ is bounded. Thus $\Lambda(x)$ can achieve its maximum only on one of the free boundaries.

Now we use the monotonicity formula to conclude that the maximum of $\Lambda(x)$ can not occur on a free boundary other than $\partial A_{n}$. To see this assume that the maximum occurs at $x_{0}$ on another free boundary $\partial\left\{\psi=t_{i}\right\}$. Let

$$
\alpha=\limsup _{\substack{y \rightarrow x_{0} \\ y \in A_{i}}}\left|\nabla \psi_{n}(y)\right|^{2}, \quad \beta=\limsup _{\substack{y \rightarrow x_{0} \\ y \in A_{i}+1}}\left|\nabla \psi_{n}(y)\right|^{2} .
$$


Using arguments from $[\mathrm{ACF}]$ one can show that the blowup limit $\tilde{\psi}$ at $x_{0}$ is a two plane solution, i.e. linear on either side of a line, with gradients $\alpha$ and $\beta$. Combining this with the monotonicity of $M(u, r), u=\psi-t_{i}$ one gets $M(\tilde{\psi}, 1) \leq M(\psi, r)$ so

$$
\frac{\alpha \beta}{4}=M(\tilde{\psi}, 1) \leq \alpha \beta \frac{\left|B_{r} \cap\left\{\psi>t_{i}\right\}\right|\left|B_{r} \cap\left\{\psi \leq t_{i}\right\}\right|}{\left|B_{r}\right|^{2}} \leq \frac{\alpha \beta}{4}
$$

Equality implies that $|\nabla \psi|$ is constant on either side of the free boundary (in some small ball) which as before leads to a contradiction due to (17). One sees again the connection between the monotonicity formula and curvature in that the monotonicity of $M(\psi, r)$ leads to the conclusion that level curves have zero curvature. To further emphasize this connection note that equality in (18) also implies that the free boundary splits $B_{r}$ exactly in two, measure wise, which would be impossible for all small $r$ at a point of nonzero curvature if the free boundary were known to be smooth. Another indication of this connection comes from the observation that one can replace the use of (17) in the above argument by an application of the monotonicity formula as above in order to show level curves have zero curvature.

In any event we have established that the maximum of $\Lambda$ (over the closure of $\Omega \backslash A_{n}$ ) occurs on $\partial A_{n}$. However one can show that the innermost free boundary $\partial A_{n}$ is convex (or consists of convex components) and that it is analytic. It then follows by free boundary calculations and a range variation that $\Lambda$ is constant, and (16) holds as an inequality, on $\partial A_{n}$. This allows us to conclude that

$$
\Lambda(x) \leq\left(\frac{\Delta t_{n}}{\left|A_{n}\right|}\right)^{2}
$$

in $\Omega$ since $\nabla \psi=0$ in $A_{n}$. But by virtue of Theorem 2 Part $\left.i i\right)$ this last quantity is bounded by the energy $J_{T}\left(\psi_{n}\right)$ of the minimizer of the $n^{\text {th }}$ approximating problem, which is bounded independently of $n$ by $\inf _{W} \tilde{J}$ $(\tilde{J}$ as in $(7))$.

Another approach to the GKK problem has been investigated by Eydeland, Lifschitz, Spruck and Turkington [EST], [LTES], [TESL]. They introduce an elegant method of approximating the variational problem (2) which is well adapted to numerical work. However they do not consider uniform gradient bounds and so cannot derive a gradient bound for minimizers of GKK (in the presence of infinitely many constraints). 


\section{References}

[ACF] H. W. Alt, L. A. Caffarelli and A. Friedman, Variational Problems with two phases and their free boundaries, Transactions AMS 282 (1984), no. 2.

[BKP] W. Becker, C. Kenig, and J. Pipher, A Convexity Property of Eigenvalues with Applications, submitted for publication.

[EST] A. Eydeland, J. Spruck, and B. Turkington, Multiconstrained variational problems of non-linear type: new formulations and algorithms, Math. Comp. 55 (1990), no. 192, 509-535.

[GHS] H. Grad, P. N. Hu and D. Stevens, Adiabatic Evolution of Plasma Equilibrium, Proc. Nat. Acad. Sci. USA 12 (1975), no. 10, 3789-3793.

[KK] M. Kruskal and R. Kulsrud, Equilibrium of a Magnetically Confined Plasma in a Toroid, Phys. of Fluids 1 (1958), no. 1, 265.

[LS1] P. Laurence and E. Stredulinsky, A New Approach to Queer Differential Equations, Comm. Pure Appl. Math. 38 (1985), 333-355.

[LS2] _ A Survey of Regularity Results for Queer Differential Equations of Second Order, Proc. Conf. in Honor of Hans Lewy, Springer Lecture Notes, vol. $3460,1986$.

[LS3] Convergence of a Sequence of Free Boundary Problems Associated to the Grad Variational Problem in Plasma Physics, Comm. Pure Appl. Math. 43 (1990), no. 4, 547-573.

[LS 4] Existence of regular solutions with convex level sets for semilinear elliptic equations with monotone $L^{1}$ nonlinearities, Part I: An approximating free boundary problem, Indiana Univ. Math. J. 39 (1990), no. 4, 1081-1114.

[LS 5] Existence of regular solutions with convex level sets for semilinear elliptic equations with nonmonotone $L^{1}$ nonlinearities, Part II: Passage to the limit, Indiana Univ. Math. J. 3 (1990), no. 2, 485-498.

[LS6] - Axisymmetric MHD equilibrium from Kruskal-Kulsrud to Grad, IMA Volumes in Mathematics and its Applications, Springer Verlag, vol. 53, 1991.

[LTES] A. Lifschitz, B. Turkington, A. Eydeland, and J. Spruck, Multiconstrained variational problems in magnetohydrodynamics, I: Equilibrium, to appear.

[M] J. Mossino, A priori estimates for a model Mercier Type in Plasma Physics, Appl. Anal. 13 (1982), no. 3, 185-207.

[MT] J. Mossino and R. Temam, Certains Problèmes non linéaires de la Physiques des Plasmas, Proc. Conf. in Rome, Springer Lect. Notes. in Math., vol. 606, 1978.

[PS] L. L. Payne and I. Stakgold, On the mean value of the fundamental mode in the fixed membrane problem, Appl. Annal. 3 (1973), 295-303.

[TESL] B. Turkington, A. Eydeland, J. Spruck, and A. Lifschitz, Multiconstrained variational problems in magnetohydrodynamics, II: slow evolution, preprint.

[W] R. White, Theory of Tokamak Plasmas, North-Holland, 1992.

Univerita di Roma "La Sapienza" Piazzale A ldo Moro 200185 , Rome italy

E-mail address: laurence@dm.unirm1.it

Univeritit of W isconsin Center-Richland Richland Center, W i 53581 ,

U.S.A. (608) 647-6225(FAX)

E-mail address: estredul@uwcmail.uwc.edu 